PROCEEDINGS OF THE

AMERICAN MATHEMATICAL SOCIETY

Volume 134, Number 5, Pages 1277-1287

S 0002-9939(05)08162-1

Article electronically published on October 18, 2005

\title{
UNIQUE FACTORIZATION IN GENERALIZED POWER SERIES RINGS
}

\author{
JAMES POMMERSHEIM AND SHAHRIAR SHAHRIARI
}

(Communicated by Carl G. Jockusch, Jr.)

\begin{abstract}
Let $K$ be a field of characteristic zero and let $K((\mathbb{R} \leq 0))$ denote the ring of generalized power series (i.e., formal sums with well-ordered support) with coefficients in $K$, and non-positive real exponents. Berarducci (2000) constructed an irreducible omnific integer, in the sense of Conway (2001), by first proving that an element of $K((\mathbb{R} \leq 0))$ that is not divisible by a monomial and whose support has order type $\omega$ (or $\omega^{\omega^{\alpha}}$ for some ordinal $\alpha$ ) must be irreducible. In this paper, we consider elements of $K((\mathbb{R} \leq 0))$ with support of order type $\omega^{2}$. The irreducibility of these elements cannot be deduced solely from the order type of their support and, after developing new tools for studying these elements, we exhibit both reducible and irreducible elements of this type. We further prove that all elements whose support has order type $\omega^{2}$ and which are not divisible by a monomial factor uniquely into irreducibles. This provides, in the ring $K((\mathbb{R} \leq 0))$, a class of reducible elements for which we have unique factorization into irreducibles.
\end{abstract}

\section{INTRODUCTION}

Let $K$ be a field and $G$ an ordered abelian group. Given a formal sum $a=$ $\sum_{\gamma} a_{\gamma} t^{\gamma}$ with coefficients $a_{\gamma}$ in $K$ and exponents $\gamma$ in $G$, the support of $a$ is the set $\operatorname{Supp}(a)=\left\{\gamma \in G \mid a_{\gamma} \neq 0\right\}$. Such a formal sum $a$ is called a generalized power series if $\operatorname{Supp}(a)$ is a well-ordered subset of $G$. The collection of generalized power series with coefficients in $K$ and exponents in $G$ is denoted by $K((G))$ and can be shown to be a field with addition and multiplication defined as for ordinary power series. We define $K\left(\left(G^{\leq 0}\right)\right)$ to consist of those elements of $K((G))$ with only non-positive exponents, i.e., $a \in K((G))$ is in $K\left(\left(G^{\leq 0}\right)\right)$ if for all $\gamma \in \operatorname{Supp}(a)$ we have $\gamma \leq 0 . K\left(\left(G^{\leq 0}\right)\right)$ is a non-noetherian integral domain, and we are interested in factorization properties of its elements.

Not every integral domain has irreducible elements. However, Berarducci [1] recently proved that if $K$ is a field of characteristic zero and $G$ is an ordered abelian divisible group, then there exist irreducible elements in $K\left(\left(G^{\leq 0}\right)\right)$. In this paper, for the case where $K$ is a field of characteristic zero and $G$ is the additive group of reals, we introduce additional tools that enable us to construct new classes of

Received by the editors January 7, 2004 and, in revised form, December 25, 2004.

2000 Mathematics Subject Classification. Primary 06F25; Secondary 13A16, 03H15, 03E10, 12J25, $13 \mathrm{~A} 05$.

Key words and phrases. Generalized power series, unique factorization, surreal numbers, omnific integers, ordered rings. 
irreducible elements. We also exhibit a collection of reducible elements that have a unique factorization into irreducibles.

For $a \in K\left(\left(G^{\leq 0}\right)\right)$ we define the order type of $a$, ot $(a)$, to be the order type of $\operatorname{Supp}(a)$. In general, ot $(a)$ is an ordinal number, and for the case when $G=\mathbb{R}$ it is a countable ordinal. When $K$ has characteristic zero, Berarducci [1 proved that if $a \in K\left(\left(\mathbb{R}^{\leq 0}\right)\right)$ is not divisible by $t^{\gamma}$ for any $\gamma<0$, and if $a$ has order type $\omega^{\omega^{\alpha}}$, then both $a$ and $a+1$ are irreducible elements of $K\left(\left(\mathbb{R}^{\leq 0}\right)\right)$. In particular, Conway's series $\sum_{n} t^{-1 / n}+1$ is irreducible.

In this paper we concentrate on elements of $K\left(\left(\mathbb{R}^{\leq 0}\right)\right)$ with order type $\omega^{2}$. We show that these elements factor uniquely into irreducibles. In addition, we exhibit collections of irreducible elements with this order type. In other words, Berarducci shows that elements whose order type is not a product of two smaller order types (and do not have a trivial factor of $t^{\gamma}$ for some $\gamma \leq 0$ ) must be irreducible. By contrast, we concentrate here on elements whose irreducibility cannot be solely deduced from their order types and we exhibit new irreducible elements of this kind. We also show that factorization into irreducibles is unique for the reducible elements with order type $\omega^{2}$.

We now state these results more precisely. Let $K$ be a field of characteristic zero and let $R=K\left(\left(\mathbb{R}^{\leq 0}\right)\right)$. In this $K$-algebra, let $J$ denote the ideal of all elements that are divisible by $t^{\gamma}$ for some $\gamma<0$, and define a $K$-vector space: $\widetilde{R}=R /(J+K)$. Now for an element $a=\sum_{\alpha} a_{\alpha} t^{\alpha} \in R$ and for $\gamma \in \mathbb{R} \leq 0$ we define the translated truncation of $a$ at $\gamma$ to be $a^{\mid \gamma}=t^{-\gamma} \sum_{\alpha \leq \gamma} a_{\alpha} t^{\alpha}$. The translated truncation of $a$ at $\gamma$ is an element of $R$, and the image of this element under the canonical homomorphism $\widetilde{\pi}: R \rightarrow \widetilde{R}$ will be called the germ of a to the left of $\gamma$ and will be denoted by $\widetilde{a^{\mid \gamma}}$. Note that the germ of $a$ to the left of $\gamma$ is zero unless $\gamma$ is a limit point of the support of $a$. Now we are ready to define our main tool in the study of elements of $R$ with order type $\omega^{2}$. Let $a \in R$ and assume that ot $(a)$ is $\omega^{2}$ or $\omega^{2}+1$. Let $V(a)$ be a subspace of $\widetilde{R}$ defined by

$$
V(a)=\operatorname{Span}_{K}\left\{\widetilde{a^{\mid \gamma}} \mid \gamma<0\right\} .
$$

Note that we can easily construct elements $a \in R$ such that $V(a)$ has arbitrarily high dimension $n$. We do this by making sure that the germs of $a$ to the left of $n$ of the limit points of the support of $a$ are linearly independent. We prove (see Corollary 3.3):

Theorem A. Let $K$ be a field of characteristic zero, $R=K\left(\left(\mathbb{R}^{\leq 0}\right)\right)$ and let $a \in R$. Assume $a \notin J+K$ and that ot $(a)$ is $\omega^{2}$ or $\omega^{2}+1$. If $\operatorname{dim}_{K}(V(a))>2$, then $a$ is irreducible.

We also produce a large class of irreducible elements with $\operatorname{dim}_{K}(V(a))=2$ (see Corollary 3.4). In addition we prove (see Theorem 4.1):

Theorem B. Let $K$ be a field of characteristic zero, $R=K((\mathbb{R} \leq 0))$ and let $a \in R$. Assume $a \notin J+K$ and that ot $(a)$ is $\omega^{2}$ or $\omega^{2}+1$. Then a has a unique factorization into irreducibles.

One motivation for studying generalized power series rings is the theory of surreal numbers developed by John Conway [2, 3, 5]. Let $G$ be the proper class No (see Conway 2] for the relevant definitions). By substituting $t^{-1}$ for $\omega$ in the normal form of a surreal number we see that Conway's ring of omnific integers is isomorphic 
to $\mathbb{R}\left(\left(G^{<0}\right)\right) \oplus \mathbb{Z}$, i.e., the elements of $\mathbb{R}\left(\left(G^{<0}\right)\right)$ with (rational) integer constant term. Berarducci [1] extended his irreducibility results appropriately and hence was able to provide the Conway series as an example of an irreducible omnific integer. Many questions about factorization properties of omnific integers remain open. Our results can be seen as providing evidence for the existence of non-trivial reducible omnific integers that factor uniquely into irreducibles.

More recently (the authors became aware of these papers after the research for the current paper was concluded), Daniel Pitteloud [6, 7] has studied rings of generalized power series and extended Berarducci's results. In particular, Pitteloud [6] has proved that if $a \in K\left(\left(\mathbb{R}^{\leq 0}\right)\right)$ has order type $\omega$ and if 0 is a limit point of support of $a$, then $a$ is prime in $K\left(\left(\mathbb{R}^{\leq 0}\right)\right)$. This result shows for the first time that the ring $K\left(\left(\mathbb{R}^{\leq 0}\right)\right)$ contains prime elements. Using this result, one can give a short proof of our Theorem $\mathrm{B}$. The direct proof of Theorem $\mathrm{B}$ that we give here is quite different. We have included this proof because it further illustrates the value of considering the vector space $V(a)$.

We refer the reader to Berarducci [1] for a list of open problems. Of particular interest to us is whether the results of this paper can be generalized to the ring $\mathbb{R}\left(\left(\mathbf{N o}^{<0}\right)\right) \oplus \mathbb{Z}$.

\section{Preliminaries}

We begin with a number of definitions related to ordinal numbers. We follow the notation and vocabulary of Berarducci [1] closely, and we refer the reader to Hrbacek and Jech [4, Chapter 7] for elementary definitions and facts about ordinals. Let $O R$ denote the class of all ordinal numbers and let $\mathbf{H}=\{\rho \in O R \mid \rho=$ 0 or $\exists \alpha \in O R$ with $\rho=\omega^{\alpha}$ \}. Elements of $\mathbf{H}$ are called additive principal since they are exactly those ordinals that cannot be written as the ordinal sum of two strictly smaller ordinals. It follows that for every ordinal number $\alpha \neq 0$, there are uniquely determined ordinals $\alpha_{1} \geq \cdots \geq \alpha_{n}$ such that $\alpha=\omega^{\alpha_{1}}+\cdots+\omega^{\alpha_{n}}$. This is called the Cantor normal form of $\alpha$, and $\omega^{\alpha_{n}}$ is called the principal part of $\alpha$.

Let $\alpha$ and $\beta$ be non-zero ordinals and consider their Cantor normal forms $\alpha=$ $\omega^{\alpha_{1}}+\ldots+\omega^{\alpha_{n}}$ and $\beta=\omega^{\alpha_{n+1}}+\ldots+\omega^{\alpha_{n+m}}$. Let $\pi$ be defined as a permutation of integers $1, \ldots, n+m$ with the property that $\alpha_{\pi(1)} \geq \cdots \geq \alpha_{\pi(n+m)}$. Now the natural sum of $\alpha$ and $\beta$ is denoted by $\alpha \oplus \beta$ and defined by $\alpha \oplus \beta=\omega^{\alpha_{\pi(1)}}+\cdots+\omega^{\alpha_{\pi(n+m)}}$. In addition for all ordinals $\alpha$, we set $0 \oplus \alpha=\alpha \oplus 0=\alpha$. The natural product of $\alpha$ and $\beta$ is denoted by $\alpha \odot \beta$ and is first defined on $\mathbf{H}$ by $\omega^{\alpha} \odot \omega^{\beta}=\omega^{\alpha \oplus \beta}$ and $\gamma \odot 0=0 \odot \gamma=0$. The definition of $\odot$ is then extended to all ordinals using the Cantor normal form and distributivity: $\gamma \odot(\alpha \oplus \beta)=(\alpha \oplus \beta) \odot \gamma=(\alpha \odot \gamma) \oplus(\beta \odot \gamma)$.

Note that the natural sum and product are commutative, unlike the usual addition and multiplication of ordinals. In addition, it is easy to show (Lemma 3.5 of Berarducci [1]) that the natural sum and product are strictly increasing, i.e., if $\alpha<\beta$, then $\alpha \oplus \gamma<\beta \oplus \gamma$, and, provided $\gamma \neq 0, \alpha \odot \gamma<\beta \odot \gamma$.

In this paper, $K$ will always denote a field of characteristic zero. Now let $R$ denote the $K$-algebra $K\left(\left(\mathbb{R}^{\leq 0}\right)\right)$. For an element $a \in R$, we often need to focus on properties of the part of the power series representing $a$ with exponents just to the left of zero. Let $J$ denote the ideal of all elements of $R$ that are divisible by $t^{\gamma}$ for some $\gamma<0$. The quotient $R / J$ will be denoted by $\bar{R}$. Note that $\bar{R}$ is an infinite-dimensional $K$-algebra. For every element $a \in R$, we define the germ of $a$ 
at zero to be the image of $a$ under the canonical homomorphism $\pi: R \rightarrow \bar{R}$. For $a \in R$, the germ of $a$ at zero will be denoted by $\bar{a} \in \bar{R}$.

Now $K$ is a subspace of $R$ and thus so is $J+K$. Recall from the introduction that $\widetilde{R}$ denotes the $K$-vector space $R /(J+K) \cong \bar{R} / \bar{K}$, and for every element $a \in R$, $\widetilde{a} \in \widetilde{R}$ denotes the image of $a$ under the canonical homomorphism $\widetilde{\pi}: R \rightarrow \widetilde{R}$. $\widetilde{a}$ is the germ of a to the left of zero. Clearly, $\widetilde{a} \neq 0$ if and only if 0 is a limit point of $\operatorname{Supp}(a)$. Also note that for $k \in K$, and $a \in R$, we have $k \bar{a}=\overline{k a}=\bar{k} \bar{a}$ while $\widetilde{k} \widetilde{a}=0 \neq \widetilde{k a}=k \widetilde{a}$ as long as $a \notin J+K$ and $k \neq 0$.

Following Berarducci, if $b=\sum_{\beta} b_{\beta} t^{\beta} \in R$ and $\gamma \in \mathbb{R}^{\leq 0}$, then we define $b_{\mid \gamma}=$ $\sum_{\beta \leq \gamma} b_{\beta} t^{\beta}$ and $b^{\mid \gamma}=t^{-\gamma} b_{\mid \gamma}$. We call $b_{\mid \gamma}$ the truncation of $b$ at $\gamma$, and $b^{\mid \gamma}$ the translated truncation of $b$ at $\gamma$. In addition, we call $\overline{b^{\mid \gamma}}$ the germ of $b$ at $\gamma$, and $\widetilde{b^{\mid \gamma}}$ the germ of $b$ to the left of $\gamma$.

The following will be useful.

Proposition 2.1 (Lemma 7.5 of Berarducci [1]). Let $b, c \in R=K((\mathbb{R} \leq 0))$ and $\gamma \in \mathbb{R} \leq 0$. Then

(1) there are only finitely many pairs $(\beta, \xi) \in \mathbb{R} \leq 0 \times \mathbb{R} \leq 0$ with $\beta+\xi=\gamma$ and $\overline{b^{\mid \beta} c^{\mid \xi}} \neq 0 \in \bar{R}$

$$
\overline{(b c)^{\mid \gamma}}=\sum_{\beta+\xi=\gamma} \overline{b^{\mid \beta}} \overline{c^{\mid \xi}} .
$$

One of the main tools used in this paper is the notion, introduced by Berarducci, of ordinal value for an element of $R=K\left(\left(\mathbb{R}^{\leq 0}\right)\right)$. For $a \in R$, the ordinal value of $a$ (at zero) is an ordinal in $\mathbf{H}$, denoted by $v(a)$ (Berarducci [1] uses $v_{J}(a)$ and Pitteloud [6] uses $v_{0}(a)$ ), and defined by:

$$
v(a)= \begin{cases}0 & \text { if } \bar{a}=0, \\ 1 & \text { i.e., } \text { if } 0 \text { is not in the closure of } \operatorname{Supp}(a), \\ & \text { if } \bar{a} \neq 0 \text { and } \widetilde{a}=0, \\ & \text { i.e., } \text { if } 0 \text { is an isolated point of } \operatorname{Supp}(a), \\ \min \{o t(b) \mid b \in R \text { and } \widetilde{b}=\widetilde{a}\} & \text { if } \widetilde{a} \neq 0, \\ & \text { i.e., } \text { if } 0 \text { is a limit point of } \operatorname{Supp}(a) .\end{cases}
$$

If $a \notin J+K$ and 0 is not in the support of $a$, then ot $(a)$ is a limit ordinal and $v(a)$ is precisely the principal part of $o t(a)$. On the other hand, if $a \notin J+K$ and $0 \in \operatorname{Supp}(a)$, then ot $(a)=\beta+1$ for some limit ordinal $\beta$ and $v(a)$ is the principal part of $\beta$. For example, if 0 is a limit point of $\operatorname{Supp}(a)$ and $o t(a)=\omega^{2} \cdot 2+\omega \cdot 5+1$, then $v(a)=\omega$. For $a \in R$ and $\gamma \in \mathbb{R}^{\leq 0}$ we call $v\left(a^{\mid \gamma}\right)$ the ordinal value of $a$ at $\gamma$. Note that, for $a=\sum_{\gamma} a_{\gamma} t^{\gamma} \in R$ and $\nu \in \mathbb{R}, v\left(a^{\mid \nu}\right)=1$ is equivalent to $\overline{a^{\mid \nu}}=\overline{a_{\nu}}$ where $a_{\nu}$ is a non-zero element of $K$. What makes the ordinal value valuable is the following multiplicative property:

Proposition 2.2 (Lemma 5.5 and Theorem 9.7 of Berarducci [1]). For $b, c \in$ $K\left(\left(\mathbb{R}^{\leq 0}\right)\right)$ we have

(a) $v(b+c) \leq \max \{v(b), v(c)\}$ with equality if $v(b) \neq v(c)$,

(b) $v(b c)=v(b) \odot v(c)$. 
Let $0 \neq b \in K((\mathbb{R} \leq 0))$. Then, by Lemma 10.1 of Berarducci [1], the set of ordinals $\left\{v\left(b^{\mid x}\right) \mid x \in \mathbb{R} \leq 0\right\}$ has a maximum $\alpha$. In addition, the set $\left\{y \mid v\left(b^{\mid y}\right) \neq 0\right\}$ is contained in the closure of the support of $b$ and hence is well ordered. A number $x \in \mathbb{R}^{\leq 0}$ is called the critical point of $b$ if $x$ is the smallest real number such that $v\left(b^{\mid x}\right)=\alpha$.

Proposition 2.3 (Lemma 10.4 of Berarducci [1]). Let $b$ and $c$ be non-zero elements of $K((\mathbb{R} \leq 0))$ with critical points $x, y$ respectively. Then

$$
v\left((b c)^{\mid x+y}\right)=v\left(b^{\mid x}\right) \odot v\left(c^{\mid y}\right) .
$$

Finally we have the main theorem of Berarducci that produces irreducible elements of $K\left(\left(\mathbb{R}^{\leq 0}\right)\right)$. The theorem basically says that if the order type of an element not in $J$ is not the product of two smaller ordinals, then the element itself is irreducible.

Theorem 2.4 (Theorem 10.5 of Berarducci [1]). Let $a \in K((\mathbb{R} \leq 0)$ ) with ot $(a)=$ $\omega^{\omega^{\alpha}}$ where $\alpha$ is an ordinal. If $\widetilde{a} \neq 0$, then $a$ and $a+1$ are irreducible elements of $K((\mathbb{R} \leq 0))$.

\section{IRREDUCIBLE ELEMENTS WITH ORDER TYPE $\omega^{2}$}

An element of $R=K\left(\left(\mathbb{R}^{\leq 0}\right)\right)$ whose order type is $\omega^{2}$ is not covered by Theorem 2.4. In this section, we use the vector space $V(a)$, defined in the introduction, to analyze the reducibility of elements of this order type.

Lemma 3.1. Let $K$ be a field of characteristic zero, $R=K((\mathbb{R} \leq 0))$, and $a \in R$. Assume $\widetilde{a} \neq 0$ and that ot $(a)$ is $\omega^{2}$ or $\omega^{2}+1$. Assume $a=b c$ where $b, c \in K((\mathbb{R} \leq 0))$ and (without loss of generality) $v(b) \leq v(c)$. Then exactly one of the following cases holds:

(1) $b \in K$ and $c=\frac{1}{b} a$ has the same order type as $a$;

(2) ot $(b)$ and ot $(c)$ are $\omega$ or $\omega+1$ and $v(b)=v(c)=\omega$.

Proof. Since $o t(a)$ is $\omega^{2}$ or $\omega^{2}+1$ and $\widetilde{a} \neq 0$, we have $v(a)=\omega^{2}$. By Proposition 2.2. we have $v(b) \odot v(c)=v(a)=\omega^{2}$. Thus either (Case 1) $v(b)=1$ and $v(c)=\omega^{2}$, or (Case 2) $v(b)=v(c)=\omega$.

Let $y$ and $z$ be critical points of $b$ and $c$ respectively. We first show that $y=z=0$. Using Proposition 2.3 and the fact that the natural product is strictly increasing, we have

$$
v\left(a^{\mid y+z}\right)=v\left(b^{\mid y}\right) \odot v\left(c^{\mid z}\right) \geq v(b) \odot v(c)=v(a)=\omega^{2} .
$$

Now the ordinal value of $a$ is greater than or equal to $\omega^{2}$ only at zero. Hence $y+z=0$ and so $y=z=0$.

For the first case, 0 is the critical point of $b$ and the ordinal value of $b$ at 0 is 1 . Hence we have $v\left(b^{\mid y}\right)=0$ for all $y<0$. This means that $b$ is an element of $K$. For the second case, where $v(b)=v(c)=\omega, 0$ is the only limit point for $b$ and $c$. Hence the order types of $b$ and $c$ are $\omega$ or $\omega+1$, and the proof is complete.

Let $K$ be a field of characteristic zero, and let $a \in R=K((\mathbb{R} \leq 0))$. Assume that $\operatorname{ot}(a)$ is $\omega^{2}$ or $\omega^{2}+1$. Define $X(a)$ to be the set of all limit points of $\operatorname{Supp}(a)$ excluding zero. In other words,

$$
X(a)=\left\{x<0 \mid v\left(a^{\mid x}\right)=\omega\right\} .
$$


Also recall from the introduction that the vector space $V(a)$ is a subspace of $\widetilde{R}$ and is defined by $V(a)=\operatorname{Span}_{K}\left\{\widetilde{a^{\mid x}} \mid x<0\right\}=\operatorname{Span}_{K}\left\{\widetilde{a^{\mid x}} \mid x \in X(a)\right\}$.

Proposition 3.2. Let $K$ be a field of characteristic zero and let $R=K((\mathbb{R} \leq 0))$. Assume ot $(a)$ is $\omega^{2}$ or $\omega^{2}+1$ and that $a=b c$ where $b=\sum_{x} b_{x} t^{x}$ and $c=\sum_{x} c_{x} t^{x}$ are elements of $R$ with $v(b)=v(c)=\omega$. Then

(1) $\tilde{a} \neq 0$, and ot $(b)$ and ot $(c)$ are $\omega$ or $\omega+1$.

(2) For $x<0$ we have

$$
\widetilde{a^{\mid x}}=c_{x} \widetilde{b}+b_{x} \widetilde{c}
$$

(3) $X(a) \subseteq \operatorname{Supp}(b) \cup \operatorname{Supp}(c) \backslash\{0\}$.

(4) $\operatorname{Supp}(b) \cup \operatorname{Supp}(c) \backslash X(a) \cup\{0\}$ is a finite subset of $\operatorname{Supp}(b) \cap \operatorname{Supp}(c)$. Moreover, if $\widetilde{b}$ and $\widetilde{c}$ are linearly independent, then $X(a)=\operatorname{Supp}(b) \cup$ $\operatorname{Supp}(c) \backslash\{0\}$.

(5) As a subspace of $\widetilde{R}, V(a)$ is spanned by $\{\widetilde{b}, \widetilde{c}\}$. In particular, $V(a)$ is at most two dimensional and is one dimensional if and only if $\widetilde{b}$ and $\widetilde{c}$ are linearly dependent elements of $\widetilde{R}$.

Proof. (1) By Proposition 2.2 we have $v(a)=v(b) \odot v(c)=\omega^{2}$. Hence $\widetilde{a} \neq 0$ and we are in the second case of Lemma 3.1. Thus the order types of $b$ and $c$ are either $\omega$ or $\omega+1$.

(2) Let $0 \neq x \in \mathbb{R} \leq 0$. By Proposition 2.1 we have

$$
\overline{a^{\mid x}}=\sum_{y+z=x} \overline{b^{\mid y}} \overline{c^{\mid z}} .
$$

On the right-hand side, $\overline{b^{\mid y}}$, the germ of $b$ at $y$, will be zero exactly when the ordinal value of $b$ at $y$ is zero. Thus on the right we only need to include terms for which the ordinal value of $b$ at $y$ and the ordinal value of $c$ at $z$ are at least 1 . On the other hand, since the order types of $b$ and $c$ are either $\omega$ or $\omega+1$, the only case where both the ordinal value of $b$ at $y$ and the ordinal value of $c$ at $z$ are greater than 1 is if $y=z=0$. This would make $x=0$, which contradicts our assumption. We thus have

$$
\overline{a^{\mid x}}=\sum_{\substack{y+z=x \\ v\left(b^{\mid y}\right)=1 \\ v\left(c^{\mid z}\right)=1}} \overline{b^{\mid y}} \overline{c^{\mid z}}+\sum_{\substack{y+z=x \\ v\left(b^{\mid y}\right)>1 \\ v\left(c^{\mid z}\right)=1}} \overline{b^{\mid y}} \overline{c^{\mid z}}+\sum_{\substack{y+z=x \\ v\left(b^{\mid y}\right)=1 \\ v\left(c^{\mid z}\right)>1}} \overline{b^{\mid y}} \overline{c^{\mid z}} .
$$

Again $v\left(b^{\mid y}\right)>1$ if and only if $y=0$ and $v\left(b^{\mid y}\right)=1$ if and only if $\overline{b^{\mid y}}=\overline{b_{y}}$. Now letting

$$
k=\sum_{\substack{y+z=x \\ v\left(b^{\mid y}\right)=1 \\ v\left(c^{\mid z}\right)=1}} b_{y} c_{z} \in K
$$

we have

$$
\begin{aligned}
\overline{a^{\mid x}} & =\bar{k}+\overline{c_{x}} \bar{b}+\overline{b_{x}} \bar{c} \\
& =\bar{k}+c_{x} \bar{b}+b_{x} \bar{c}
\end{aligned}
$$

So

$$
\widetilde{a^{\mid x}}=c_{x} \widetilde{b}+b_{x} \widetilde{c}
$$


(3) If $x \in X(a)$, then $\widetilde{a^{\mid x}} \neq 0$, and hence by the previous part we cannot have both $c_{x}$ and $b_{x}$ equal to zero. This means that $x \in \operatorname{Supp}(b) \cup \operatorname{Supp}(c)$. The conclusion follows since $0 \notin X(a)$.

(4) If $x \notin X(a) \cup\{0\}$, then $v\left(a^{\mid x}\right)$ is 0 or 1 , and in either case $\widetilde{a^{\mid x}}=0$. By the previous part this means that $c_{x} \widetilde{b}+b_{x} \widetilde{c}=0$. Now if either $c_{x}$ or $b_{x}$ is not zero, then they are both non-zero since neither $\widetilde{b}$ nor $\widetilde{c}$ is zero. Also if $\widetilde{b}$ and $\widetilde{c}$ are linearly independent, then $c_{x}=b_{x}=0$ and $x \notin \operatorname{Supp}(b) \cup \operatorname{Supp}(c)$. It remains to show that if $\widetilde{b}$ and $\widetilde{c}$ are linearly dependent, then $\operatorname{Supp}(b) \cup \operatorname{Supp}(c) \backslash X(a) \cup\{0\}$ is a finite set. So assume $\widetilde{c}=k \widetilde{b}$ for some $0 \neq k \in K$ and let $x \in \operatorname{Supp}(b) \cup \operatorname{Supp}(c) \backslash X(a) \cup\{0\}$. Then, as was argued above, $c_{x} \widetilde{b}+b_{x} \widetilde{c}=0$ and neither $b_{x}$ nor $c_{x}$ is zero. Hence $\widetilde{c}=\frac{-c_{x}}{b_{x}} \widetilde{b}$. Thus $\frac{-c_{x}}{b_{x}}=k$, and we conclude that $c_{x}=-k b_{x}$ for all $x \in \operatorname{Supp}(b) \cup$ $\operatorname{Supp}(c) \backslash X(a) \cup\{0\}$. On the other hand $\widetilde{c}=k \widetilde{b}$ implies that $c=k b+j+k^{\prime}$ where $j \in J$ and $k^{\prime} \in K$. Since the order type of $c$ is $\omega$ or $\omega+1, j$ has finite support and thus we conclude that $c$ and $k b$ differ in at most a finite number of terms. Thus $c_{y}=k b_{y}$ except for possibly a finite number of terms. The conclusion now follows.

(5) By (2), it is clear that $V(a) \subseteq \operatorname{span}_{K}\{\widetilde{b}, \widetilde{c}\}$. Now if $\widetilde{b}$ and $\widetilde{c}$ are linearly dependent, then $\operatorname{span}_{K}\{\widetilde{b}, \widetilde{c}\}$ is a one-dimensional vector space and hence cannot be strictly larger than $V(a)$. So assume that $\widetilde{b}$ and $\widetilde{c}$ are linearly independent.

If $y_{0}$ and $z_{0}$ are respectively the least elements of $\operatorname{Supp}(b)$ and $\operatorname{Supp}(c)$, then $x_{0}=y_{0}+z_{0}$ is the least element of $\operatorname{Supp}(a), a_{x_{0}}=b_{y_{0}} c_{z_{0}}$, and we have $\frac{1}{a_{x_{0}}} a=$ $\left(\frac{1}{b_{y_{0}}} b\right)\left(\frac{1}{c_{z_{0}}} c\right)$. Hence without loss of generality we can assume that $a_{x_{0}}=b_{y_{0}}=$ $c_{z_{0}}=1$ and $y_{0} \leq z_{0}$. Now since $z_{0}$ is the least element of $\operatorname{Supp}(c)$, we have $c_{y_{0}}=0$ if $y_{0}<z_{0}$, and $c_{y_{0}}=c_{z_{0}}=1$ if $y_{0}=z_{0}$.

Now to obtain a contradiction, we will assume that $\operatorname{dim} V(a)=1$. It then follows that $\widetilde{a^{\mid y_{0}}}=c_{y_{0}} \widetilde{b}+\widetilde{c}$ spans $V(a)$. Let $y$ be an arbitrary element of $\operatorname{Supp}(b) \cup$ $\operatorname{Supp}(c) \backslash\{0\}$. By part (44), $\widetilde{a^{\mid y}} \neq 0$ and so $\widetilde{a^{\mid y}}=k\left(c_{y_{0}} \widetilde{b}+\widetilde{c}\right)$ for some non-zero $k \in K$. By part (2), we have

$$
\widetilde{a^{\mid y}}=c_{y} \widetilde{b}+b_{y} \widetilde{c} .
$$

Since $\{\widetilde{b}, \widetilde{c}\}$ is linearly independent, we conclude

$$
\begin{aligned}
& c_{y}=k c_{y_{0}}, \\
& b_{y}=k .
\end{aligned}
$$

Now if $c_{y_{0}}=0$, then $c_{y}=0$ for all $y \in \operatorname{Supp}(c) \backslash\{0\}$, which would make $\widetilde{c}=0$, a contradiction. So $c_{y_{0}}=1$ and we have $c_{y}=k=b_{y}$. We conclude that $b-b_{0}=a-a_{0}$ and hence $\widetilde{b}=\widetilde{c}$, yet another contradiction, which completes the proof.

Using Proposition 3.2 we can immediately identify a number of irreducibility criteria for generalized power series with real exponents that are of order type $\omega^{2}$ or $\omega^{2}+1$ and have zero as a limit point of their support. The first result is Theorem A of the introduction.

Corollary 3.3. Let $K$ be a field of characteristic zero, let $R=K\left(\left(\mathbb{R}^{\leq 0}\right)\right)$ and let $a \in R$. Assume $\widetilde{a} \neq 0$ and that ot $(a)$ is $\omega^{2}$ or $\omega^{2}+1$. If $\operatorname{dim}_{K}(V(a))>2$, then $a$ is irreducible.

Proof. This is immediate from Lemma (3.1) and part (5) of Proposition 3.2 . 
Corollary 3.4. Let $K$ be a field of characteristic zero, let $R=K\left(\left(\mathbb{R}^{\leq 0}\right)\right)$ and let $a \in R$. Assume $\widetilde{a} \neq 0$ and that ot $(a)$ is $\omega^{2}$ or $\omega^{2}+1$. Assume $\operatorname{dim}_{K}(V(a))=2$ and that there exists an element $\alpha \in \operatorname{Supp}(a)$ that is not the sum of two elements of $X(a)$. Then a is irreducible.

Proof. If $a$ is reducible, then by Proposition 3.1, $a=b c$ where $v(b)=v(c)=\omega$. $\alpha \in \operatorname{Supp}(a)$ and so $\alpha$ is the sum of an element of $\operatorname{Supp}(b)$ and an element of $\operatorname{Supp}(c)$. By part (5) of Proposition 3.2, $\widetilde{b}$ and $\widetilde{c}$ are linearly independent and hence by part (4) of Proposition 3.2, $X(a)=\operatorname{Supp}(b) \cup \operatorname{Supp}(c) \backslash\{0\}$. Thus, contrary to the assumption, $\alpha$ has to be the sum of two elements from $X(a)$. The contradiction shows that $a$ is irreducible.

\section{UNIQUE FACTORIZATION FOR ELEMENTS OF ORDER TYPE $\omega^{2}$}

In the ring $R=K((\mathbb{R} \leq 0))$, it is easy to construct reducible elements of order type $\omega^{2}$ (for example $\left.\left(\sum_{n=1}^{\infty} t^{-1 / n}\right)^{2}\right)$ and we have seen families of irreducible elements with this order type. In this section we prove Theorem $B$ of the introduction, which states that elements with this order type having zero as a limit point of their support factor uniquely into irreducibles. The proof is independent of Pitteloud's proof [6] that elements with order type $\omega$ that have zero as a limit point of their support are prime in $R$. We use a linear algebraic argument based on the vector space $V(a)$.

Theorem 4.1. Let $K$ be a field of characteristic zero, let $R=K\left(\left(\mathbb{R}^{\leq 0}\right)\right)$ and let $a \in R$. Assume $\widetilde{a} \neq 0$ and that ot $(a)$ is $\omega^{2}$ or $\omega^{2}+1$. Then a has a unique factorization into irreducibles.

Proof. If $a$ is irreducible, then there is nothing to prove and otherwise, by Lemma 3.1 $a=b c$ where $o t(b)$ and $o t(c)$ are $\omega$ or $\omega+1$ and $v(b)=v(c)=\omega$. By Theorem 2.4 both $b$ and $c$ are irreducible and hence $a$ is a product of irreducibles. It remains to show that this factorization is unique (up to units). Thus assume $b c=a=d e$ where the order types of $b, c, d$ and $e$ are $\omega$ or $\omega+1$ and the ordinal value of each of these is $\omega$. By Corollary $3.3, \operatorname{dim} V(a) \leq 2$. We complete the proof in two cases depending on the dimension of this vector space.

Assume $\operatorname{dim} V(a)=1$. By Proposition 3.2, any one of $\widetilde{b}, \widetilde{c}, \widetilde{d}$, or $\widetilde{e}$ provides a basis for $V(a)$ and hence there exist $k_{1}, k_{2}, k_{3} \in K$ with

$$
\widetilde{c}=k_{1} \widetilde{b}, \quad \widetilde{d}=k_{2} \widetilde{b}, \quad \widetilde{e}=k_{3} \widetilde{b} .
$$

Pulling back to the ring $R$, there exist $k_{4}, k_{5}, k_{6} \in K$ and $j_{1}, j_{2}, j_{3} \in J$ such that

$$
\begin{aligned}
c & =k_{1} b+j_{1}+k_{4}, \\
d & =k_{2} b+j_{2}+k_{5}, \\
e & =k_{3} b+j_{3}+k_{6} .
\end{aligned}
$$

Note that since 0 is the only limit point of $\operatorname{Supp}(c), \operatorname{Supp}(d)$, and $\operatorname{Supp}(e)$ we conclude that $j_{1}, j_{2}$ and $j_{3}$ have finite order types. Substituting the corresponding expressions in $b c=d e$ we get

$$
b[A+B]=C
$$


where

$$
\begin{aligned}
& A=\left(k_{1}-k_{2} k_{3}\right) b, \\
& B=j_{1}-k_{2} j_{3}-k_{3} j_{2}+k_{4}-k_{2} k_{6}-k_{3} k_{5}, \\
& C=k_{5} k_{6}+k_{5} j_{3}+k_{6} j_{2}+j_{2} j_{3} .
\end{aligned}
$$

By Proposition 2.2, $v(C)=v(b) \odot v(A+B)=\omega \odot v(A+B)$. Now $v(C)<\omega$ since $C \in J+K$ and so we conclude that the only possibility is for $v(C)=v(A+B)=0$. Thus $C \in J$ and so $k_{5} k_{6}=0$. By part (a) of Proposition 2.2, $v(A+B)=\omega$ if $k_{1} \neq k_{2} k_{3}$. It follows that $k_{1}=k_{2} k_{3}$ and $B \in J$. So we also have $k_{4}=k_{2} k_{6}+k_{3} k_{5}$. The equation $b[A+B]=C$ now becomes $b B=C$ where

$$
\begin{aligned}
& B=j_{1}-k_{2} j_{3}-k_{3} j_{2}, \\
& C=k_{5} j_{3}+k_{6} j_{2}+j_{2} j_{3} .
\end{aligned}
$$

Assume $B \neq 0$ and let the critical point of $B$ be at $y$ where $y \leq 0$. Since $B$ and $C$ have finite order types, we have that $v\left(B^{\mid y}\right)$ and $v\left(C^{\mid y}\right)$ are no greater than 1 . The critical point of $b$ is at zero, and using Proposition 2.3 we get

$$
1 \geq v\left(C^{\mid y}\right)=v(b) \odot v\left(B^{\mid y}\right)=\omega \odot v\left(B^{\mid y}\right) .
$$

Since the natural product is strictly increasing, the only possibility is if $v\left(B^{\mid y}\right)=0$. But this is impossible if $y$ is the critical point of $B$. Hence $B=0$ and it follows that $C=0$ as well.

Summarizing the above we now have

$$
\begin{aligned}
k_{5} k_{6} & =0, \\
k_{1}-k_{2} k_{3} & =0, \\
k_{4}-k_{2} k_{6}-k_{3} k_{5} & =0, \\
j_{1}-k_{2} j_{3}-k_{3} j_{2} & =0, \\
k_{5} j_{3}+k_{6} j_{2}+j_{2} j_{3} & =0 .
\end{aligned}
$$

Without loss of generality, we can assume $k_{5}=0$. Then we have

$$
\begin{aligned}
k_{5} & =0, \\
k_{1}-k_{2} k_{3} & =0, \\
k_{4}-k_{2} k_{6} & =0 \\
j_{1}-k_{2} j_{3}-k_{3} j_{2} & =0 \\
j_{2}\left(k_{6}+j_{3}\right) & =0 .
\end{aligned}
$$

Since $R$ is an integral domain, $j_{2}$ or $k_{6}+j_{3}$ must be zero. In the former case, we get that $c=k_{2} e$ and $d=k_{2} b$. In the latter, we get that $c=k_{3} d$ and $e=k_{3} b$. In either case $d$ and $e$ are associates of $b$ and $c$ and the proof of the first case is complete.

For the second case, assume $\operatorname{dim} V(a)=2$. By Proposition 3.2, $\{\widetilde{b}, \widetilde{c}\}$ and $\{\widetilde{d}, \widetilde{e}\}$ form two bases for the vector space $V(a)$. Thus there exists an invertible $2 \times 2$ matrix $P$ with entries in $K$ such that in $\widetilde{R}$ we have

$$
\left[\begin{array}{l}
\widetilde{d} \\
\widetilde{e}
\end{array}\right]=P\left[\begin{array}{l}
\widetilde{b} \\
\widetilde{c}
\end{array}\right] .
$$

By Proposition 3.2 we have that for any $x<0$ we have

$$
b_{x} \widetilde{c}+c_{x} \widetilde{b}=\widetilde{a^{\mid x}}=d_{x} \widetilde{e}+e_{x} \widetilde{d} .
$$


We can write this as

$$
\left[\begin{array}{ll}
c_{x} & b_{x}
\end{array}\right]\left[\begin{array}{l}
\widetilde{b} \\
\widetilde{c}
\end{array}\right]=\left[\begin{array}{ll}
e_{x} & d_{x}
\end{array}\right]\left[\begin{array}{l}
\widetilde{d} \\
\widetilde{e}
\end{array}\right]=\left[\begin{array}{ll}
e_{x} & d_{x}
\end{array}\right] P\left[\begin{array}{l}
\widetilde{b} \\
\widetilde{c}
\end{array}\right] .
$$

Now since $\{\widetilde{b}, \widetilde{c}\}$ is a linearly independent set, we must have that for all $x<0$,

$$
\left[\begin{array}{ll}
c_{x} & b_{x}
\end{array}\right]=\left[\begin{array}{ll}
e_{x} & d_{x}
\end{array}\right] P .
$$

Thus except possibly for the constant term, every term of the generalized power series $b$ and $c$ is a fixed linear combination of the terms of $d$ and $e$. Hence there exist constants $k_{b}, k_{c} \in K$ such that

$$
\left[\begin{array}{ll}
c & b
\end{array}\right]=\left[\begin{array}{ll}
e & d
\end{array}\right] P+\left[\begin{array}{ll}
k_{c} & k_{b}
\end{array}\right] .
$$

Moding out by $J+K$ and passing to $\widetilde{R}$ we have

$$
\left[\begin{array}{ll}
\widetilde{c} & \widetilde{b}
\end{array}\right]=\left[\begin{array}{ll}
\widetilde{e} & \widetilde{d}
\end{array}\right] P .
$$

Putting (11) and (3) together, we get

$$
\left[\begin{array}{ll}
\widetilde{b} & \widetilde{c}
\end{array}\right] P^{t}\left[\begin{array}{ll}
0 & 1 \\
1 & 0
\end{array}\right]=\left(P\left[\begin{array}{l}
\widetilde{b} \\
\widetilde{c}
\end{array}\right]\right)^{t}\left[\begin{array}{ll}
0 & 1 \\
1 & 0
\end{array}\right]=\left[\begin{array}{ll}
\widetilde{e} & \widetilde{d}
\end{array}\right]=\left[\begin{array}{ll}
\widetilde{c} & \widetilde{b}
\end{array}\right] P^{-1} .
$$

Now let $P=\left[\begin{array}{ll}\alpha & \beta \\ \gamma & \delta\end{array}\right]$, let $\Delta=\operatorname{det}(P)$, and substitute this into the last equation to get

$$
\left[\begin{array}{ll}
\widetilde{b} & \widetilde{c}
\end{array}\right]\left[\begin{array}{ll}
\gamma & \alpha \\
\delta & \beta
\end{array}\right]=\frac{1}{\Delta}\left[\begin{array}{ll}
\widetilde{c} & \widetilde{b}
\end{array}\right]\left[\begin{array}{cc}
\delta & -\beta \\
-\gamma & \alpha
\end{array}\right] .
$$

Equating entries and using the fact that $\{\widetilde{b}, \widetilde{c}\}$ is linearly independent we get that there are only two possibilities:

$$
P=\left[\begin{array}{ll}
\alpha & 0 \\
0 & \delta
\end{array}\right] \quad \text { with } \quad \alpha \delta=1 \quad \text { or } \quad P=\left[\begin{array}{ll}
0 & \beta \\
\gamma & 0
\end{array}\right] \quad \text { with } \quad \beta \gamma=1 .
$$

The analysis for both cases is the same and so without loss of generality assume $P$ is diagonal with determinant one. Using equation (2) we get

$$
\begin{aligned}
& c=\alpha e+k_{c}, \\
& b=\delta d+k_{b} .
\end{aligned}
$$

From $b c=d e$ we get $\delta k_{c} d+\alpha k_{b} e+k_{b} k_{c}=0$ and passing to $\widetilde{R}$, we have

$$
\delta k_{c} \widetilde{d}+\alpha k_{b} \widetilde{e}=0 .
$$

However, $\{\widetilde{d}, \widetilde{e}\}$ is linearly independent in $\widetilde{R}$ and hence $k_{b}=k_{c}=0$. We conclude that $c=\alpha e$ and $b=\delta d$, and the proof is complete.

\section{REFERENCES}

[1] A. Berarducci. Factorization in generalized power series. Transactions of the American Mathematical Society, 352(2):553-577, 2000. MR.1473431 (2000c:13033)

[2] J. H. Conway. On Numbers and Games. A K Peters, Ltd., Natick, Massachusetts, second edition, 2001. MR 1803095 (2001j:00008)

[3] H. Gonshor. An Introduction to the Theory of Surreal Numbers. Number 110 in London Mathematical Society Lecture Note Series. Cambridge University Press, Cambridge, 1986. MR0872856 (88b:04002)

[4] K. Hrbacek and T. Jech. Introduction to Set Theory. Number 85 in Monographs and Textbooks in Pure and Applied Mathematics. Marcel Dekker, Inc., New York and Basel, second edition, 1984. MR0758796 (85m:04001) 
[5] D. E. Knuth. Surreal Numbers. Addison-Wesley Publishing Company, Reading, Massachusetts, 1974. MR0472278 (57:11983)

[6] D. Pitteloud. Existence of prime elements in rings of generalized power series. The Journal of Symbolic Logic, 66(3):1206-1216, September 2001. MR1856737 (2002f:13047)

[7] D. Pitteloud. Algebraic properties of rings of generalized power series. Annals of Pure and Applied Logic, 116:39-66, 2002. MR1900901 (2003f:13022)

Department of Mathematics, Reed College, Portland, Oregon 97202

E-mail address: jamie@reed.edu

Department of Mathematics, Pomona College, Claremont, California 91711

E-mail address: sshahriari@pomona.edu 